\title{
Magnetopause reconnection rate estimates for Jupiter's magnetosphere based on interplanetary measurements at $\sim 5 \mathrm{AU}$
}

\author{
J. D. Nichols ${ }^{1}$, S. W. H. Cowley ${ }^{1}$, and D. J. McComas ${ }^{2}$ \\ ${ }^{1}$ Department of Physics \& Astronomy, University of Leicester, Leicester LE1 7RH, UK \\ ${ }^{2}$ Southwest Research Institute, San Antonio, Texas, USA
}

Received: 13 October 2005 - Accepted: 1 December 2005 - Published: 7 March 2006

\begin{abstract}
We make the first quantitative estimates of the magnetopause reconnection rate at Jupiter using extended in situ data sets, building on simple order of magnitude estimates made some thirty years ago by Brice and Ionannidis (1970) and Kennel and Coroniti $(1975,1977)$. The jovian low-latitude magnetopause (open flux production) reconnection voltage is estimated using the Jackman et al. (2004) algorithm, validated at Earth, previously applied to Saturn, and here adapted to Jupiter. The high-latitude (lobe) magnetopause reconnection voltage is similarly calculated using the related Gérard et al. (2005) algorithm, also previously used for Saturn. We employ data from the Ulysses spacecraft obtained during periods when it was located near $5 \mathrm{AU}$ and within $5^{\circ}$ of the ecliptic plane (January to June 1992, January to August 1998, and April to October 2004), along with data from the Cassini spacecraft obtained during the Jupiter flyby in 2000/2001. We include the effect of magnetospheric compression through dynamic pressure modulation, and also examine the effect of variations in the direction of Jupiter's magnetic axis throughout the jovian day and year. The intervals of data considered represent different phases in the solar cycle, such that we are also able to examine solar cycle dependency. The overall average low-latitude reconnection voltage is estimated to be $\sim 230 \mathrm{kV}$, such that the average amount of open flux created over one solar rotation is $\sim 500 \mathrm{GWb}$. We thus estimate the average time to replenish Jupiter's magnetotail, which contains $\sim 300$ $500 \mathrm{GWb}$ of open flux, to be $\sim 15-25$ days, corresponding to a tail length of $\sim 3.8-6.5 \mathrm{AU}$. The average high-latitude reconnection voltage is estimated to be $\sim 130 \mathrm{kV}$, associated with lobe "stirring". Within these averages, however, the estimated voltages undergo considerable variation. Generally, the low-latitude reconnection voltage exhibits a "background" of $\sim 100 \mathrm{kV}$ that is punctuated by one or two significant enhancement events during each solar rotation, in which the voltage is elevated to $\sim 1-3 \mathrm{MV}$. The high-latitude voltages are estimated to be about a half of these values. We
\end{abstract}

Correspondence to: J. D. Nichols

(jdn@ion.le.ac.uk) note that the peak values of order a few MV are comparable to the potential drop due to sub-corotating plasma flows in the equatorial magnetosphere between $\sim 20 \mathrm{R}_{\mathrm{J}}$ and the magnetopause, such that during these periods magnetopause reconnection may have a significant effect on the otherwise rotationally dominated magnetosphere. Despite such variations during each solar rotation, however, the total amount of open flux produced during each solar rotation varies typically by less than $\sim 30 \%$ on either side of the overall average for that epoch. The averages over individual data epochs vary over the solar cycle from $\sim 600 \mathrm{GWb}$ per solar rotation at solar maximum to $\sim 400 \mathrm{GWb}$ at solar minimum. In addition we show that the IMF sector with positive clock angle is favoured for reconnection when the jovian spin axis clock angle is also positive, and vice versa, although this effect represents a first order correction to the voltage, which is primarily modulated by IMF strength and direction.

Keywords. Magnetospheric physics (Electric fields; Planetary magnetospheres; Solar wind-magnetosphere interactions)

\section{Introduction}

It is well known that the dynamics of Jupiter's near-planet magnetosphere are dominated by the internal energy source of planetary rotation, rather than by the solar wind as is the case at the Earth, coupled with the production, transport, and loss of plasma from the highly productive Io source (e.g. Hill, 1979; Pontius, 1997, Vasyliunas 1983; Delamere and Bagenal, 2003). The tapping of the huge energy reservoir that is Jupiter's rotation is spectacularly manifest in the magnetosphere-ionosphere coupling current system that is associated with the breakdown of corotation of iogenic plasma and the formation of the main auroral oval (Cowley and Bunce, 2001; Hill 2001; Khurana, 2001; Southwood and Kivelson, 2001). This dominance of corotational flow over that of the Dungey cycle driven by the solar wind is traditionally illustrated by a comparison of the magnitudes 
of the equatorial electric fields associated with each flow, which for Jupiter are directed radially outward and duskdawn respectively (Brice and Ioannidis, 1970; Kennel and Coroniti, 1975, 1977). These authors used "typical" values of the solar wind parameters based on Pioneer-10 and -11 data to estimate a Dungey cycle voltage of $\sim 1 \mathrm{MV}$, compared this with a corotation voltage of order $\sim 400 \mathrm{MV}$, and concluded that the dominant regime was that of corotation.

To quote Kennel and Coroniti (1975) in this context, however, "one neglects reconnection at his peril". The dominance of corotation does not preclude there being a significant solar wind interaction as well, as is manifest in the existence of a substantial magnetic tail that extends at least $\sim 4$ AU downstream of Jupiter, to the orbit of Saturn. For example, Lepping et al. (1983) presented evidence to suggest that Saturn was within Jupiter's tail during the Voyager-2 encounter. In addition to the existence of an extended magnetotail, HST images of Jupiter's aurora show features located at higher latitudes than the main auroral oval that have been interpreted as being due to magnetic reconnection occurring at the dayside magnetopause (e.g. Pallier and Prangé, 2001, 2003; Grodent et al., 2003, 2004; Bunce et al., 2004). In a recent study, Nichols and Cowley (2005) used a realistic middle magnetosphere current sheet field along with a theoretically computed equatorial plasma angular velocity profile, and showed that the vast majority of the above corotation potential falls within $\sim 20 \mathrm{R}_{\mathrm{J}}$ of the planet. They calculated the potential difference between $20 \mathrm{R}_{\mathrm{J}}$ and $60 \mathrm{R}_{\mathrm{J}}$ to be only $\sim 4 \mathrm{MV}$. Cowley et al. (2005) recently expanded this work to include the outer magnetosphere region and computed the voltage between $\sim 20 \mathrm{R}_{\mathrm{J}}$ and the magnetopause to be $\sim 10 \mathrm{MV}$. Thus, the magnetopause reconnection estimate of $\sim 1 \mathrm{MV}$ is clearly not negligible in this region. Reconnection-related flows may not therefore always be negligible within Jupiter's magnetosphere, though they will be confined to its outer regions. However, magnetopause reconnection is expected to be strongly modulated by the magnitude and orientation of the interplanetary magnetic field (IMF), such that one might expect associated modulation of these flows, as well as of the dynamics of the jovian cusp, and the tail regions (e.g. Acuña et al. 1983; Nishida and Maezawa, 1986). The rate of dayside reconnection may therefore have important implications for other tail processes, such as the loss of iogenic plasma down-tail in the Vasyliunas cycle (Vasyliunas, 1983).

Save for the simple order of magnitude estimates of Brice and Ioannidis (1970) and Kennel and Coroniti (1975, 1977), however, to date no systematic study of the magnitude of jovian magnetopause reconnection exists. In this paper we address this issue using the extended in situ data now available. Specifically, we examine Ulysses and Cassini data from intervals when the spacecraft were located near Jupiter's orbit near the ecliptic plane at $\sim 5 \mathrm{AU}$, these intervals also allowing us to sample various phases of the solar cycle. We begin in Sect. 2 with a discussion of the data intervals used in this study, and follow in Sect. 3 with a description of the empirical formulas we employ to estimate the reconnection- associated voltages. We then present our results in Sect. 4, followed by a discussion of the results and summary in Sect. 5.

\section{Data intervals}

The monitoring (or the lack thereof) of the interplanetary medium upstream of Jupiter is a continuing problem for planetary magnetospheric physicists. Without knowledge of the condition of the solar wind and IMF as it impinges on Jupiter's magnetosphere it is almost impossible to infer the effect of the former on the latter. However, there exists significant data concerning the interplanetary medium in the vicinity of Jupiter's orbit. In this paper we use data from the Ulysses and Cassini spacecraft, both of which have made flybys of Jupiter, in 1992 and 2000/2001 respectively. However, since 1992, Ulysses has completed two orbits over the poles of the Sun and has hence passed through the region of Jupiter's orbit twice more, in 1998 and 2004, though Jupiter itself was not then in the vicinity of the spacecraft. Thus, if one considers "Jupiter space" to be the region near Jupiter's orbit in which one may sample solar wind conditions that are similar to those which Jupiter itself experiences, we can also use two further intervals of data. The semi-major axis, eccentricity, and inclination of Jupiter's orbit are $\sim 5.2 \mathrm{AU}, \sim 0.048$, and $\sim 1.3^{\circ}$, respectively (Fränz and Harper, 2002). Hence Jupiter's heliocentric distance varies between $\sim 5.0$ and $5.4 \mathrm{AU}$, and its orbit is modestly inclined to the J2000 ecliptic plane. Here we define "Jupiter space" to be the region within $5^{\circ}$ heliocentric latitude of the $\mathrm{J} 2000$ ecliptic plane, and with a heliocentric range between 5.0 and 5.4 AU. Ulysses' orbit is such that it reaches aphelion of $\sim 5.4 \mathrm{AU}$ near to $0^{\circ}$ heliocentric latitude, hence the latter restriction on heliocentric range is automatically satisfied for the spacecraft. We therefore consider Ulysses data over the following periods - from January to June 1992, January to August 1998, and April to October 2004. Cassini data from November and December of 2000 were also employed, limited to this period due to the somewhat incomplete data coverage in the relevant region (discussed further below). In order to place these intervals in the context of the solar cycle, we show in Fig. 1 a plot of 25-day averaged sunspot number, obtained from the Solar Influences Data Analysis Centre (SIDC), Belgium, against time from 1991 to mid-2005, along with grey boxes which denote the intervals considered. It can be seen that the intervals cover various phases in the solar cycle, such that we may expect the nature of the solar wind to vary accordingly (e.g. McComas et al., 2001). Studies at Earth (e.g. Hapgood et al., 1991) have shown that the strength of the IMF does vary systematically over the solar cycle, with the magnitude of the field being $50 \%$ higher at solar maximum than at solar minimum. These variations should therefore be reflected in the reconnection voltages. The Ulysses 1992 interval samples solar maximum/beginning declining phase, and would be expected to be characterised, therefore, by high field strengths. The 


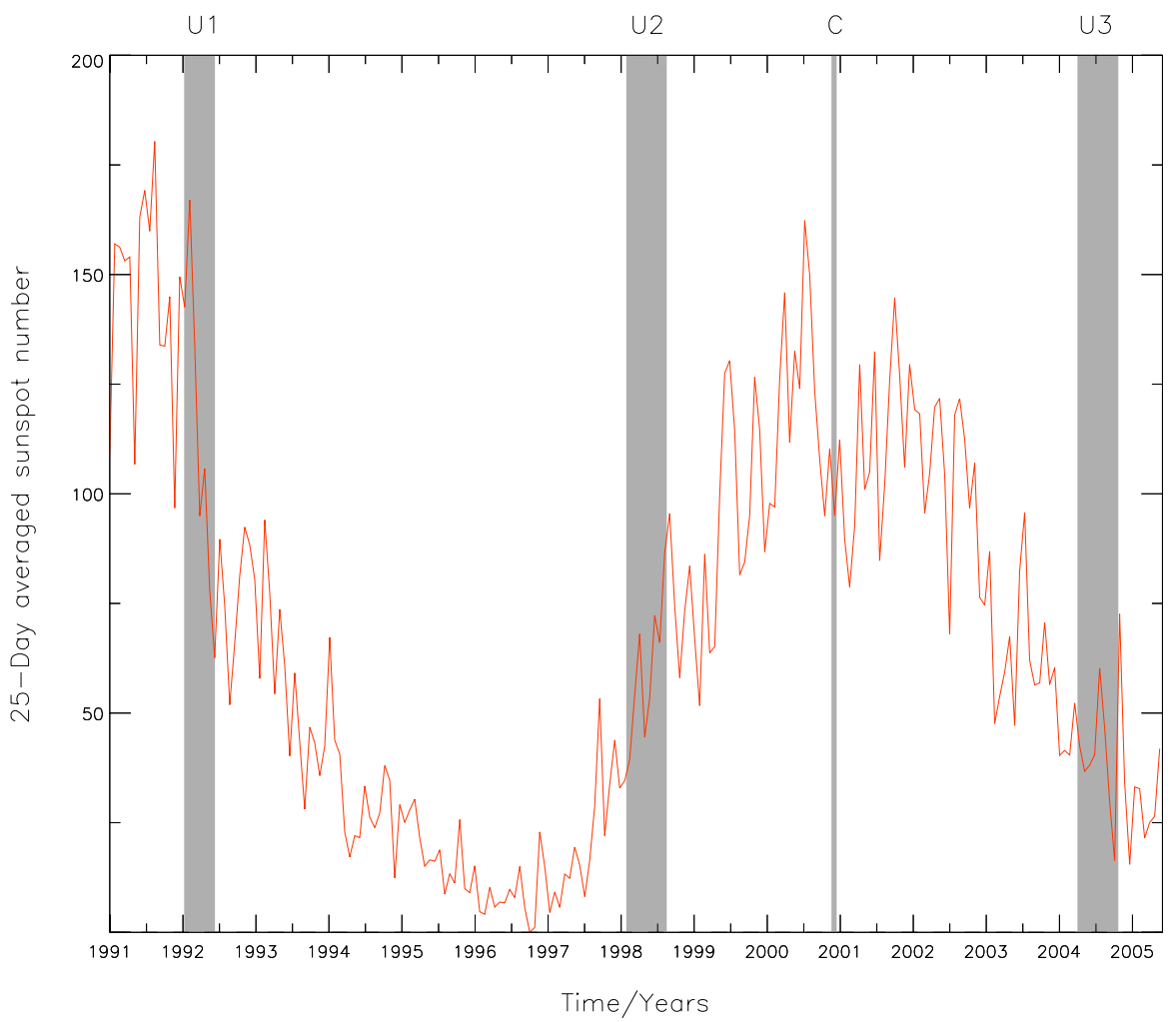

Fig. 1. Plot showing the 25-day averaged sunspot number versus time from 1991 to mid-2005. The grey boxes indicate the intervals of spacecraft data considered in this paper, marked $\mathrm{U}$ or $\mathrm{C}$ for Ulysses and Cassini respectively.

Ulysses 1998 interval lies in the middle of the rising phase, such that we would expect the field strengths to be moderately less than in the 1992 interval. The Cassini millennium flyby took place during solar maximum, such that, again, we may expect high field strengths. Finally, the Ulysses 2004 interval samples declining phase/solar minimum, such that the field strengths would be expected to be relatively low with respect the other intervals. Overall, therefore, this study samples a range of solar wind conditions, such that we would expect to observe a corresponding variation in the reconnection voltage deduced.

\section{Calculation of the reconnection voltage}

In this section we will develop empirical formulas, based on terrestrial experience, which allow us to estimate the reconnection voltages at Jupiter. We concentrate mainly on the "low-latitude" reconnection voltage, $\phi_{L}$, equal to the rate of open flux production, which is significant for the flux content and dynamics of the tail. We also consider, however, the "high-latitude" or lobe reconnection voltage, $\phi_{H}$, which does not change the amount of open flux in the system if reconnection occurs at one lobe only for a given interplanetary field line, as will generally be the case. However, lobe reconnection does result in the circulation of flux within the open field region, and may influence high-latitude precipitation and auroral patterns.
The general formula for calculating the magnetopause reconnection voltage $\phi$ for any planet, equal to the rate of open flux production, is $\phi=v_{s w} B_{\perp} L$. Here $v_{s w}$ is the velocity of the solar wind, $B_{\perp}$ is the magnitude of the perpendicular component of the IMF (such that $v_{s w} B_{\perp}$ is the interplanetary electric field), and $L$ is the width of the solar wind channel, perpendicular to $B_{\perp}$, for which reconnection occurs with the planetary field. As stated in the introduction, presently available estimates of the low-latitude jovian magnetopause reconnection voltage stem from the studies of Brice and Ioannidis (1970) and Kennel and Coroniti $(1975,1977)$, who assumed that $L=\beta L_{M}$, where $L_{M}$ is the "width" of the magnetosphere, and $\beta$ is a "reconnection efficiency parameter" assumed on the basis of terrestrial experience to be $\sim 0.1$. For example, Kennel and Coroniti (1977) employed "canonical" values of $v_{s w}=400 \mathrm{~km} \mathrm{~s}^{-1}$ and $B_{\perp}=1 \mathrm{nT}$ based on Pioneer-10 and -11 data, along with a "width" of the magnetosphere of $L_{M}=100 \pi \mathrm{R}_{\mathrm{J}}$, to obtain a value of $\sim 1 \mathrm{MV}$.

Today, thirty years later, the theory of reconnection is not yet developed to a stage where ab initio calculations of the jovian magnetopause reconnection voltage can be made. However, numerous studies at Earth using spacecraft and radar data have established clear empirical relationships between interplanetary parameters and reconnection voltage data that we can reasonably scale and apply to other planets. Here we employ an empirical formula for the voltage that was first developed and validated for the Earth (Perrault and 


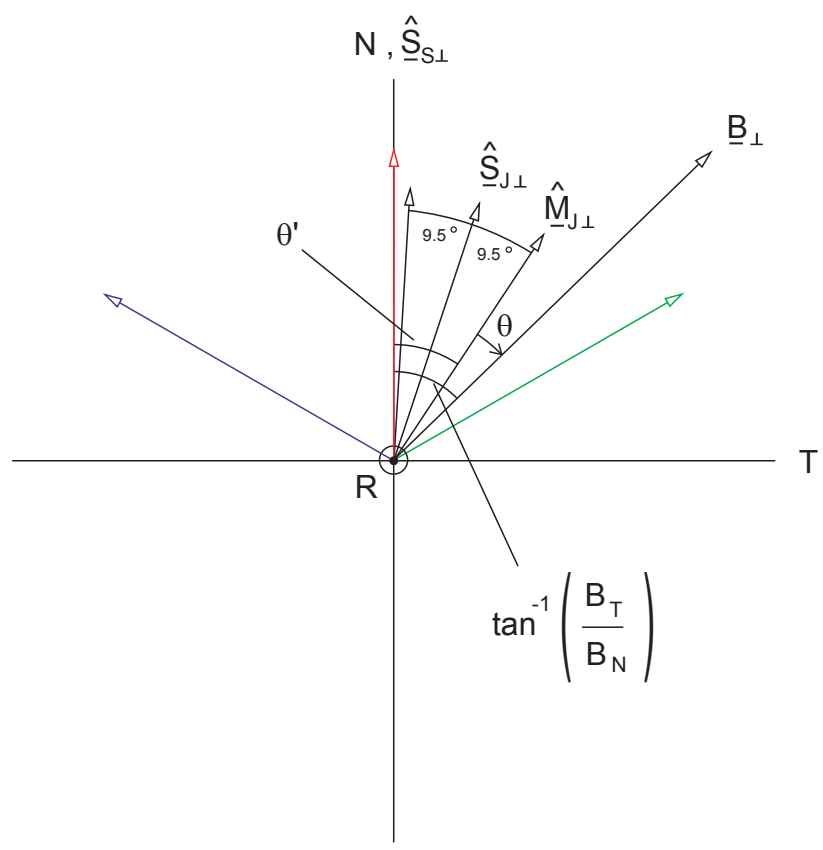

Fig. 2. Schematic diagram showing the vectors relevant to the calculation of the reconnection voltage, projected into the plane perpendicular to the Sun-Jupiter direction (i.e. the T-N plane). The Sun's spin vector $\hat{S}_{S \perp}$, also representing the N-axis, points vertically upwards, such that the T-axis points to the right, and the outward radial axis $\mathrm{R}$ points out of the page. Angles about the R-axis are measured clockwise from the $\mathrm{N}$-axis. Jupiter's spin and magnetic axes are shown by the vectors labelled $\hat{S}_{J \perp}$ and $\hat{M}_{J \perp}$. As indicated, each jovian day $\hat{\boldsymbol{M}}_{\boldsymbol{J}} \perp$ undergoes an excursion about $\hat{\boldsymbol{S}}_{\boldsymbol{J}}$ through an angle essentially equal to the $\sim 9.5^{\circ}$ offset between the magnetic axis and the spin axis. The maximum positive and negative excursions of $\hat{S}_{J \perp}$ as Jupiter orbits the Sun are shown (exaggerated) by the green and blue vectors respectively (the amplitude is actually $\sim 7.9^{\circ}$ ), while the red vector shows the case when $\hat{\boldsymbol{S}}_{\boldsymbol{J}}$ is

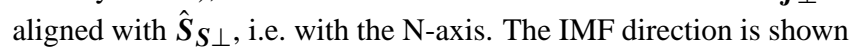
by the long vector labelled $\boldsymbol{B}_{\perp}$. The clock angle of the field $\theta$, i.e. the angle between $\hat{\boldsymbol{M}}_{\boldsymbol{J} \perp}$ and $\boldsymbol{B}_{\perp}$, is taken to be positive clockwise as shown.

Akasofu, 1978; Milan et al., 2004), and was then adapted for use at Saturn by Jackman et al. (2004). This expression for the low-latitude dayside reconnection voltage, equal to the rate of open flux production, is given by

$\phi_{L}=v_{s w} B_{\perp} L_{\mathrm{o}} \cos ^{4}(\theta / 2)$,

such that the width of the solar wind channel in the above discussions is given by $L=L_{\mathrm{o}} \cos ^{4}(\theta / 2)$, where $\theta$ is the "clock" angle between the IMF vector and Jupiter's magnetic axis projected onto a plane perpendicular to the Sun-Jupiter direction. Here we do not attempt to map the observed solar wind conditions to the planet, which would clearly be inappropriate for the 1998 and 2004 epochs, but simply calculate the voltage as though Jupiter was present at the spacecraft, as will be discussed further below. The calculation is, in principal, similar to that of Kennel and Coroniti $(1975,1977)$.
However, here we employ an extended time series of data rather than just "spot" values, and can thus examine the variations that occur over each solar rotation, from one rotation to another, and over the solar cycle.

With regard to the parameter $L_{\mathrm{o}}$ in Eq. (1), Milan et al. (2004) concluded that an appropriate empirical value for the Earth is $L_{\mathrm{o}} \sim 5 R_{E}$, i.e. approximately half the subsolar radius of the planet's magnetosphere. This result was used by Jackman et al. (2004) to scale the formula for use at Saturn, and, in the absence of a model tailored specifically for the jovian case, the same logic is now applied here as a reasonable estimate for Jupiter. Here we therefore take $L_{\mathrm{o}}$ to be half the subsolar radius of Jupiter's magnetosphere $R_{m p}$, a value which is dependent on the dynamic pressure of the solar wind. Huddleston et al. (1998) give an empirical formula for this dependence, that is

$$
R_{m p}=\frac{35.5}{p_{d y n}(\mathrm{nPa})^{0.22}} \mathrm{R}_{\mathrm{J}},
$$

where $p_{d y n}(\mathrm{nPa})$ is the dynamic pressure of the solar wind in $\mathrm{nPa}$, given by

$p_{d y n}=\rho v_{s w}^{2}$,

where $\rho$ is the mass density of the solar wind plasma, derived from the number density $n_{s w}$ by multiplication by the mean solar wind plasma particle mass.

The function $\cos ^{4}(\theta / 2)$ represents the modulation of the rate of reconnection due to the direction of the IMF. It should be noted that the function is such that it is equal to 1 when $\theta=0^{\circ}, 0.25$ when $\theta= \pm 90^{\circ}$, and 0 when $\theta=180^{\circ}$, such that reconnection occurs most rapidly when the IMF is directed northward (recall that the polarity of Jupiter's magnetic field is opposite to that of the Earth's), and shuts off when the IMF is southward. Most commonly, however, the IMF lies near the equatorial plane, such that the prevailing clock angles are $\pm 90^{\circ}$, depending on the IMF sector. As with the $L_{\mathrm{o}}$ parameter, this function of $\theta$ represents a reasonable estimate for the jovian case, given experience at Earth and the absence of knowledge to the contrary at Jupiter.

Here we also employ a related formula for the highlatitude reconnection voltage. Specifically we calculate the high-latitude reconnection voltage using Gérard et al.'s (2005) adaptation of the Jackman et al. (2004) expression for low-latitude reconnection, given by

$\phi_{H}=\frac{1}{2} v_{s w} B_{\perp} L_{\mathrm{o}} \sin ^{4}(\theta / 2)$.

In this expression the sin function has replaced the cos dependence in Eq. (1), indicating its association with southward IMF, and the magnitude is estimated to be approximately half that of the low-latitude reconnection voltage (see Gerard et al. (2005) and Bunce et al. (2005) for further information).

We now consider the calculation of the "clock" angle $\theta$ from the magnetic data. The coordinate system in which the magnetometer data for both Ulysses and Cassini are rendered is the RTN system. This is defined such that $\mathrm{R}$ points radially 
away from the Sun, $\mathrm{N}$ is perpendicular to this axis and is such that the R-N plane contains the Sun's spin axis vector, and T then forms the right-handed set, pointing generally in the direction of planetary motion. Figure 2 shows a schematic of the relevant vectors and angles projected onto the T-N plane (hence the " $\perp$ " labels on the vectors), looking towards the Sun. Note that clock angles about the R-axis are measured clockwise from the $\mathrm{N}$-axis. The Sun's projected spin axis $\hat{S}_{S \perp}$, which also represents the $\mathrm{N}$-axis, points vertically upwards, such that the T-axis points to the right. Jupiter's spin axis projected into the T-N plane, $\hat{S}_{J \perp}$, then lies at some angle to $\hat{\boldsymbol{S}}_{S \perp}$ dependent on Jupiter's orbital longitude. Over the course of Jupiter's orbit around the Sun, $\hat{S}_{J \perp}$ moves between angles of $\sim-7.9^{\circ}$ and $\sim+7.9^{\circ}$ relative to the $\mathrm{N}$-axis, represented in Fig. 2 by the blue and green vectors respectively (the effect being considerably exaggerated in the diagram for sake of clarity). The red vector then represents the case where $\hat{S}_{S \perp}$ and $\hat{S}_{J \perp}$ are co-aligned along the $\mathrm{N}$-axis in the T-N plane. Jupiter's magnetic axis is offset from the spin axis by $\sim 9.5^{\circ}$, such that it oscillates around the spin axis by this amount over the course of a jovian day. When projected onto the T-N plane, this offset angle is only modified in the second decimal place for all spin axis angles, such that, as indicated in the figure, we just take the angle $\theta^{\prime}$ between the Sun's projected spin vector $\hat{\boldsymbol{S}}_{\boldsymbol{S} \perp}$ and Jupiter's magnetic axis vector $\hat{\boldsymbol{M}}_{\boldsymbol{J} \perp}$ to be the spin axis clock angle plus and minus $9.5^{\circ}$. The clock angle of the field, i.e. the angle between $B_{\perp}$ and $\hat{\boldsymbol{M}}_{\boldsymbol{J} \perp}$, as shown in Fig. 2, is thus given by

$\theta=\tan ^{-1}\left(\frac{B_{T}}{B_{N}}\right)-\theta^{\prime}$,

where $B_{T}$ and $B_{N}$ are the $T$ and $N$ components of the IMF. We also have in terms of these components

$B_{\perp}=\sqrt{B_{T}^{2}+B_{N}^{2}}$.

From any given IMF data point we thus derive a range of voltages due both to the effect of Jupiter's orbital motion, shown in Fig. 2 by the red, green, and blue vectors, and its rotation, shown in the figure by the range of $\hat{\boldsymbol{M}}_{J \perp}$ around $\hat{S}_{J \perp}$. The effect of the direction of $\hat{\boldsymbol{M}}_{J \perp}$ on the derived reconnection rates can be readily understood from Fig. 2. Suppose, for example, the angle of $\hat{\boldsymbol{M}}_{\boldsymbol{J}} \perp$ relative to the $\mathrm{N}$-axis is negative, towards the direction of the blue vector. Then if $B_{\perp}$ points in the $-\mathrm{T}$ direction it will have a component northward relative to $\hat{\boldsymbol{M}}_{\boldsymbol{J} \perp}$ and reconnection will be favoured. If, on the other hand, $B_{\perp}$ points in the + T direction it will have a southward component relative to $\hat{M}_{J \perp}$, and reconnection will be disfavoured. The opposite applies when $\hat{\boldsymbol{M}}_{J \perp}$ points toward the green vector, of course. We should note, however, that these spin and magnetic axis effects represent only first order corrections to the voltage, which, as will be seen, is dominated by the large variations in the IMF magnitude and direction at Jupiter.

The quantities thus required from the spacecraft are the $B_{T}$ and $B_{N}$ components of the IMF obtained from the Ulysses FGM/VHM (Balogh et al., 1992) and Cassini MAG
(Dougherty et al., 2004) magnetometers, and $n_{s w}$ and $v_{s w}$, obtained from the Ulysses SWOOPS (Bame et al., 1992) and Cassini CAPS (Young et al., 2004) plasma particle detectors. We have averaged these data over ten minute intervals, and linearly interpolated between data gaps. This latter comment is most pertinent to the Cassini CAPS data, as the instrument was limited in its ability to make observations during the Jupiter flyby due to spacecraft pointing restrictions.

\section{Results}

Each interval of interplanetary data analysed here covers a number of months, such that it is not feasible to reproduce the data set in its entirety. Instead we give in Table 1 the values of the parameters occurring in Eq. (1) averaged over each solar rotation and each epoch, i.e. $B_{\perp}, \cos ^{4}(\theta / 2), v_{s w}, R_{m p}(=2 L)$, and $\phi_{L}$, along with $\phi_{H}$ and the total open flux produced during each solar rotation $\Phi$. For the Ulysses data one solar rotation is chosen to be the interval between two successive zeroes of Carrington longitude (approximately 25 Earth days), while for Cassini we chose the 25-day interval that had the most continuous data coverage from the magnetic field and plasma instruments. In Figs. 3-6 we also show one solar rotation of typical data from 1992, 1998, 2000, and 2004, respectively. The upper panels show the "data ingredients" of the calculated parameters, while the lower panels show the results. Thus, from top to bottom we have $B_{\perp}$ in nT from Eq. (6), $\theta$ in degrees from Eq. (5), $\cos ^{4}(\theta / 2), v_{s w}$ in $\mathrm{km} \mathrm{s}^{-1}, n_{s w}$ in $\mathrm{cm}^{-3}, p_{d y n}$ in $\mathrm{nPa}$ from Eq. (3) (note the log scale in the panels for these last two quantities), $R_{m p}$ in $\mathrm{R}_{\mathrm{J}}$ from Eq. (2), $\phi_{L}$ in $\mathrm{kV}$ from Eq. (1), $\phi_{H}$ in $\mathrm{kV}$ from Eq. (4), and finally $\Phi$ in GWb obtained by integrating $\phi_{L}$ over time from zero at the start of each interval. Parameters that depend on the IMF clock angle $\theta$, i.e. $\theta$ itself, $\cos ^{4}(\theta / 2), \phi_{L}$, $\phi_{H}$ and $\Phi$, are shown with the jovian spin and magnetic axis variations described above taken into account. That is, each "data point" is stretched into a vertical bar whose extent represents the range of voltage values due to the $\pm 9.5^{\circ}$ diurnal variation of Jupiter's magnetic axis vector. In addition, the orbital effect is included using the same colour scheme as used in Fig. 2. Thus the blue trace represents the voltage for the maximum negative angle of Jupiter's spin axis relative to the $\mathrm{N}$-axis, green represents the voltage for the maximum positive angle, and red, which is plotted over the top of the blue and green traces where they overlap, represents the case where Jupiter's spin axis is aligned with the $\mathrm{N}$-axis (and thus with the Sun's spin axis in the T-N plane). We note that the averaged values quoted below and in Table 1 correspond to the middle of the red trace.

We first consider the 1992 Ulysses data which represents solar maximum/beginning declining phase conditions. The interval we examine spans days 008-161, although we omit one solar rotation of data containing the magnetosphere flyby, such that we consider five solar rotations of data. An example of this data is shown in Fig. 3 covering the solar rotation during days $085-110$. The features in the magnetic 
Table 1. Table summarising the parameters occurring in Eqs. (1) and (4) for the low- and high-latitude magnetopause reconnection voltages over the intervals examined. Each value is the average for the particular solar rotation, except $\Phi$ which is the total amount of open flux produced over the rotation period (i.e. the time integral of $\phi_{L}$ ). The averages over each epoch are shown in bold. The intervals in italics are those used as illustrative examples in this paper. Note that one solar rotation is omitted from the 1992 Ulysses data, corresponding to the jovian magnetosphere flyby.

\begin{tabular}{|c|c|c|c|c|c|c|c|c|}
\hline Epoch & Days & $B_{\perp} / \mathrm{nT}$ & $\cos ^{4}(\theta / 2)$ & $v_{s w} / \mathrm{km} \mathrm{s}^{-1}$ & $R_{m p}(=2 L) / \mathrm{R}_{\mathrm{J}}$ & $\phi_{L} / \mathrm{kV}$ & $\phi_{H} / \mathrm{kV}$ & $\Phi / \mathrm{GWb}$ \\
\hline & $008-033$ & 1.21 & 0.38 & 502 & 58 & 372 & 248 & 801 \\
1992 & $059-085$ & 1.04 & 0.34 & 474 & 62 & 293 & 173 & 643 \\
Ulysses & $085-110$ & 0.68 & 0.35 & 436 & 63 & 265 & 93 & 582 \\
& $110-135$ & 0.99 & 0.33 & 416 & 63 & 260 & 156 & 571 \\
& $135-161$ & 1.03 & 0.28 & 429 & 59 & 236 & 176 & 519 \\
& Average & $\mathbf{1 . 0 0}$ & $\mathbf{0 . 3 0}$ & $\mathbf{4 6 1}$ & $\mathbf{6 0}$ & $\mathbf{2 7 9}$ & $\mathbf{1 6 6}$ & $\mathbf{6 1 1}$ \\
\hline & $024-050$ & 0.53 & 0.34 & 375 & 65 & 144 & 68 & 316 \\
& $050-075$ & 0.72 & 0.36 & 373 & 69 & 240 & 90 & 528 \\
& $075-101$ & 0.82 & 0.30 & 373 & 66 & 249 & 99 & 546 \\
Ulysses & $101-126$ & 0.71 & 0.32 & 411 & 68 & 224 & 113 & 493 \\
& $126-151$ & 0.70 & 0.32 & 493 & 69 & 262 & 125 & 575 \\
& $151-177$ & 0.95 & 0.32 & 435 & 63 & 306 & 154 & 672 \\
& $177-202$ & 0.61 & 0.37 & 467 & 76 & 228 & 98 & 501 \\
& $202-228$ & 0.83 & 0.31 & 415 & 68 & 215 & 167 & 471 \\
& Average & $\mathbf{0 . 7 3}$ & $\mathbf{0 . 3 3}$ & $\mathbf{4 1 8}$ & $\mathbf{6 8}$ & $\mathbf{2 3 4}$ & $\mathbf{1 1 4}$ & $\mathbf{5 1 3}$ \\
\hline Cassini & $323-348$ & 0.83 & 0.30 & 489 & 59 & 223 & 128 & 483 \\
\hline & $092-118$ & 0.64 & 0.30 & 468 & 74 & 212 & 113 & 466 \\
& $118-143$ & 0.63 & 0.30 & 422 & 73 & 180 & 105 & 396 \\
& $143-168$ & 0.57 & 0.30 & 426 & 72 & 161 & 92 & 353 \\
Ulysses & $168-194$ & 0.58 & 0.32 & 435 & 71 & 197 & 84 & 433 \\
& $194-219$ & 0.61 & 0.27 & 445 & 72 & 162 & 130 & 355 \\
& $219-245$ & 0.66 & 0.33 & 471 & 72 & 151 & 166 & 331 \\
& $245-270$ & 0.74 & 0.38 & 458 & 74 & 265 & 137 & 583 \\
& $270-295$ & 0.48 & 0.38 & 386 & 72 & 174 & 62 & 382 \\
& Average & $\mathbf{0 . 6 1}$ & $\mathbf{0 . 3 2}$ & $\mathbf{4 3 9}$ & $\mathbf{7 3}$ & $\mathbf{1 8 8}$ & $\mathbf{1 1 1}$ & $\mathbf{4 1 2}$ \\
\hline
\end{tabular}

field and plasma data are generally consistent with the occurrence of corotating interaction regions (CIRs), which are known to be a dominant feature of the solar wind in the vicinity of Jupiter's orbit (e.g. Gosling and Pizzo, 1999; Gazis, 2000; Kunow, 2001), and are associated with heliospheric current sheet (HCS) crossings. It can be seen in the clock angle panel that in this interval the IMF exhibits essentially a two-sector structure (the sectors being identified by their respective $\pm 90^{\circ}$ prevailing clock angles), with a crossing of the HCS occurring somewhere between day 90 and day 97, during which interval of generally elevated field strengths there is seen to be considerable structure in the magnetic field and plasma parameters. The clock angle of the IMF is a major factor in the modulation of the rate of magnetopause reconnection. This dependency is illustrated in the third panel, which shows the factor $\cos ^{4}(\theta / 2)$ that appears in Eq. (1) and varies between one and zero depending on the IMF direction, as discussed above. The relative values of the different colours (spin axis orientations) will be discussed below. As indicated above, the HCS crossing is accompanied by a corresponding enhancement in the field magnitude to $\sim 2$ nT between days 90-97. During this period the plasma number density shown in the fifth panel is highly variable, ranging between $\sim 0.05$ and $\sim 2 \mathrm{~cm}^{-3}$, indicating significant embedded structure within the compression region. The re- gion of enhanced field is then followed by a rarefaction region, in which the field strength is low (a few tenths of a $\mathrm{nT}$ ), the plasma velocity steadily decreases over time from $\sim 500 \mathrm{~km} \mathrm{~s}^{-1}$ to $\sim 400 \mathrm{~km} \mathrm{~s}^{-1}$, and the number density is relatively stable at $\sim 0.2 \mathrm{~cm}^{-3}$. A second HCS crossing appears to occur on day 108 , accompanied by a second interval of enhanced field strengths that begins approximately one day later, the beginning of which can be just seen at the end of the interval shown. The dynamic pressure, and hence the estimated subsolar magnetopause distance, shown in the sixth and seventh panels, reflect the large changes in the number density, such that the subsolar magnetopause distance ranges between $\sim 40$ and $\sim 100 \mathrm{R}_{\mathrm{J}}$ in the vicinity of the first HCS crossing during days 90-97, and is reasonably constant at $\sim 60 \mathrm{R}_{\mathrm{J}}$ during the rarefaction region.

The bottom three panels of Fig. 3, which are the most important in terms of this paper, then show the reconnection voltages calculated from these parameters using Eqs. (1) and (4), and the amount of open flux produced since the start of the interval. The low-latitude reconnection voltage that occurs in the rarefaction region has a "background" value of the order $\sim 100 \mathrm{kV}$, consistent with a field strength of $\sim 0.2 \mathrm{nT}$, a plasma velocity of $\sim 450 \mathrm{~km} \mathrm{~s}^{-1}$, a subsolar magnetopause distance of $\sim 60 \mathrm{R}_{\mathrm{J}}$, and a prevailing IMF clock angle of $90^{\circ}$. During this solar rotation, however, there was one 


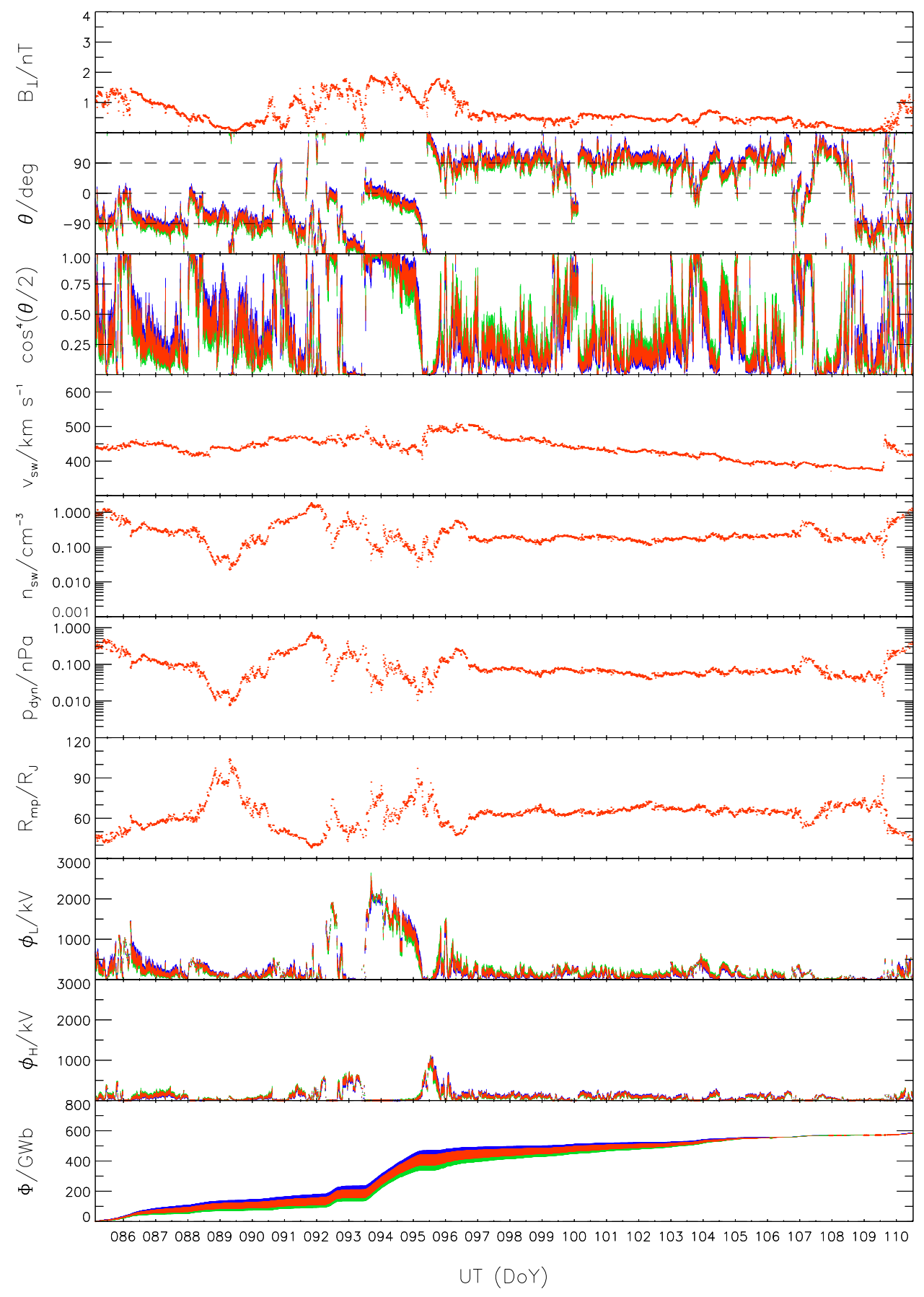

Fig. 3. Plot showing the "ingredients" of the models for the reconnection voltages from Ulysses data over days $85-110$ in 1992 , together with the estimated voltages and integrated open flux production. From top to bottom the panels show $B_{\perp}$ in nT given by Eq. (6), $\theta$ in degrees given by Eq. (5), $v_{s} w$ in $\mathrm{km} \mathrm{s}^{-1}, n_{s w}$ in $\mathrm{cm}^{-3}$ on a log scale, $p_{d y n}$ given by Eq. (3) in $\mathrm{nPa}$ on a log scale, $R_{m p}$ in $\mathrm{R}_{\mathrm{J}}$ given by Eq. (2), $\phi_{L}$ in $\mathrm{kV}$ given by Eq. (1), $\phi_{H}$ in $\mathrm{kV}$ given by Eq. (4), and finally $\Phi$ in GWb calculated by integrating $\phi_{L}$ over time, starting from zero at the initial time. In the plots for $\theta, \phi_{L}, \phi_{H}$, and $\Phi$ each "data point" is stretched into a vertical line representing the effect produced by the $\sim \pm 9.5^{\circ}$ diurnal variation of Jupiter's dipole axis offset. In addition, the blue, green, and red traces represent differing orientations of Jupiter's spin axis relative to the Sun's spin axis during the jovian orbit, as shown in Fig. 2. 
$\begin{array}{ll}\text { Ulysses FGM/VHM and SWOOPS data } & 1998 \text { Days 075-101 }\end{array}$

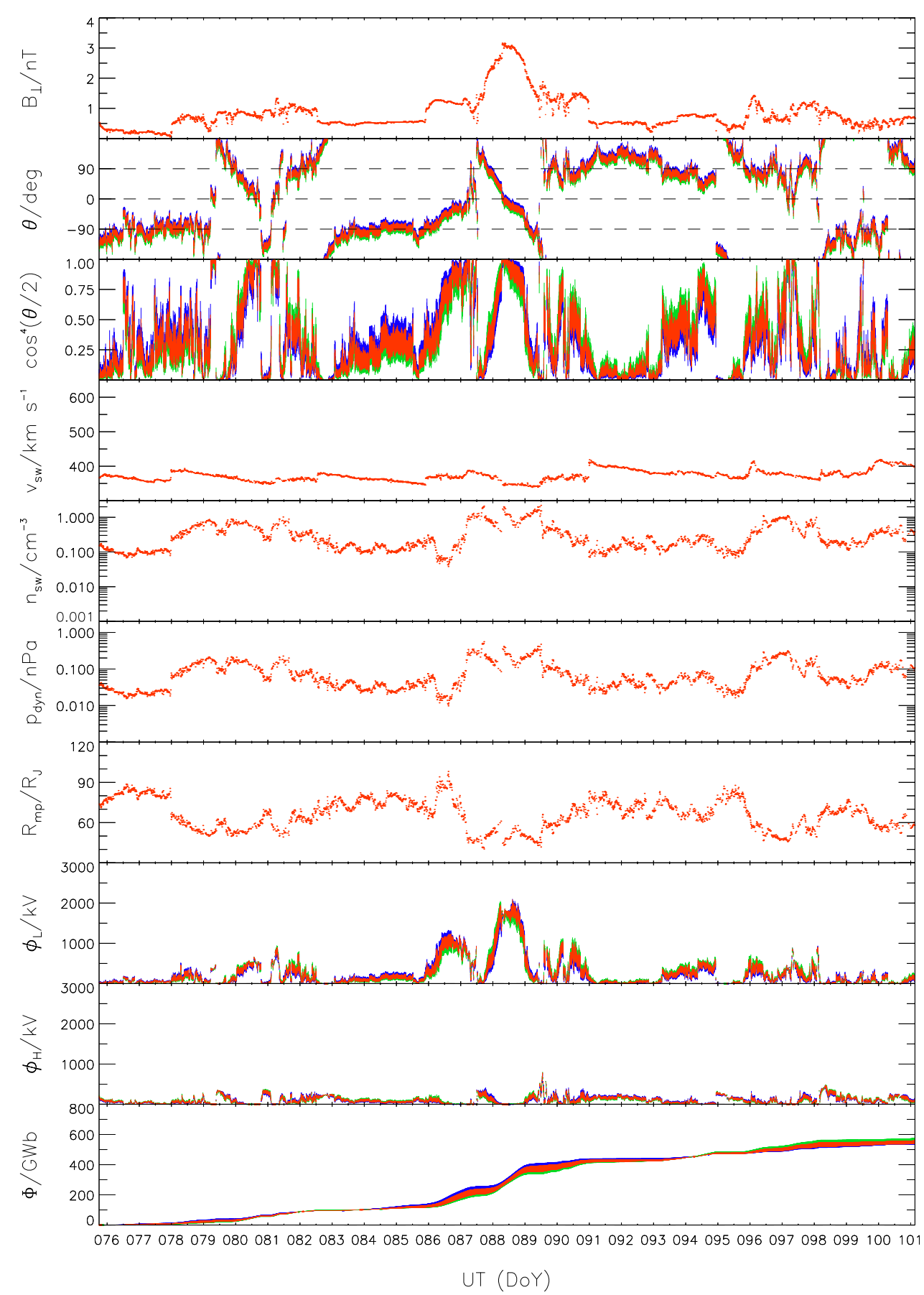

Fig. 4. As Fig. 3 for Ulysses data from the interval over days 75-101 in 1998.

significant period of enhanced voltage values associated with the region of high strength, often northward, IMF between days 90 and 97, in which the estimated low-latitude voltage peaked at $\sim 2.4 \mathrm{MV}$. In this period the high-latitude voltage also peaked at $\sim 1 \mathrm{MV}$ where the IMF briefly turned southward. It can be seen in the bottom panel that the total open flux estimated to have been produced over this solar rotation is $\sim 580 \mathrm{GWb}$, corresponding to an average low-latitude voltage of $\sim 265 \mathrm{kV}$ over the interval. However, it should be noted that $\sim 350 \mathrm{GWb}$, over half of the total estimated to have been produced over the solar rotation, is produced between days 90 and $97, \sim 200 \mathrm{GWb}$ of which is created during days 93 and 94 alone. 


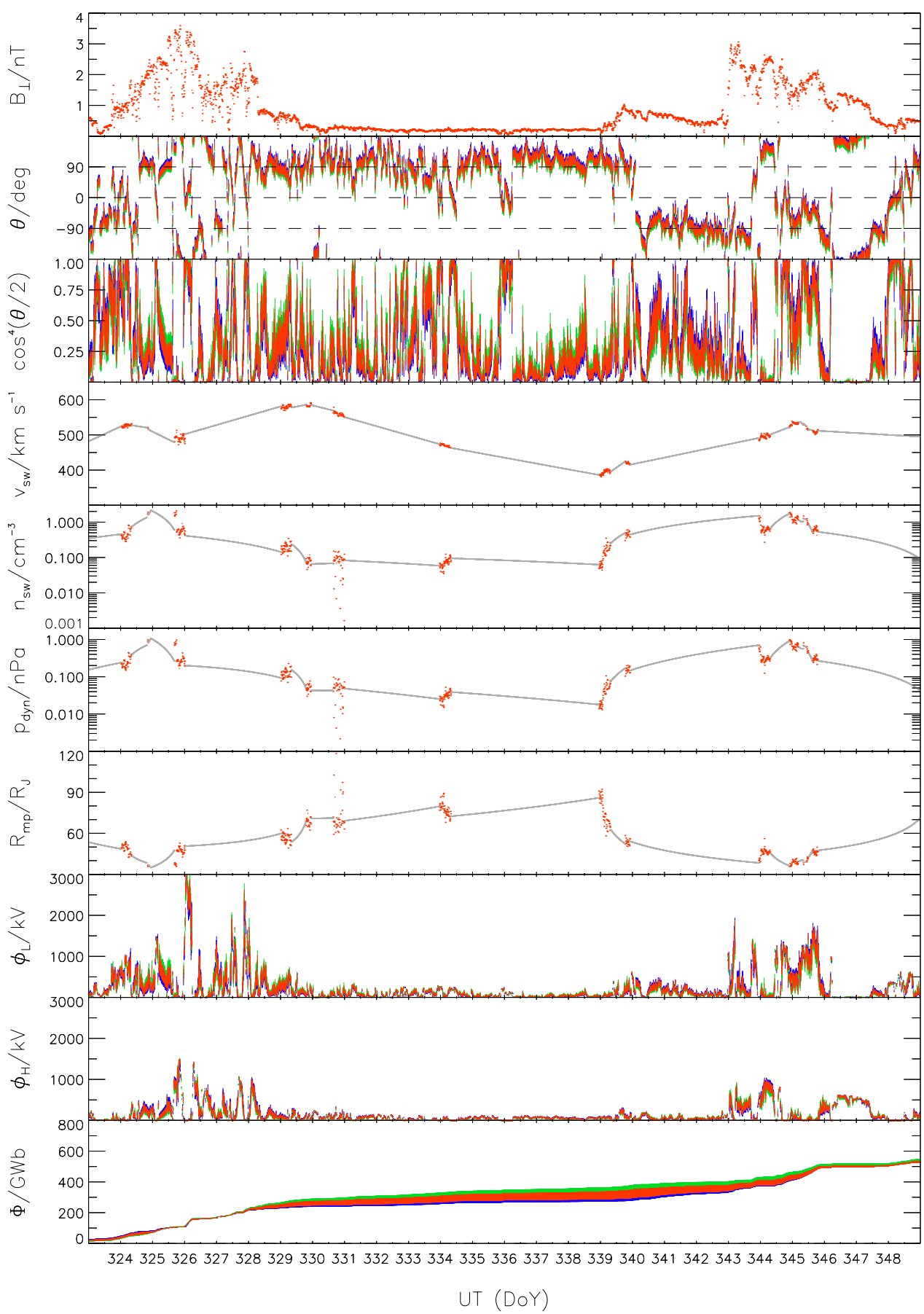

Fig. 5. As Fig. 3 for Cassini data from the interval over days 323-348 in 2000.

With regard to the spin and magnetic axis effects on the rate of reconnection, the former can be seen in the difference between the variously coloured data in the $\theta, \cos ^{4}(\theta / 2), \phi_{L}$, $\phi_{H}$ and $\Phi$ panels, while the latter can be seen in the vertical extent of the data bars (although as the red bars are plotted over the blue and green where they overlap, only the full width of the red trace can be seen). Thus during the predominantly negative $B_{T}$ sector $\left(\theta \simeq-90^{\circ}\right)$ before day $\sim 90$, the blue trace, representing the case where the spin axis is at its maximum negative angle to the $\mathrm{N}$-axis (see Fig. 2), exhibits higher $\cos ^{4}(\theta / 2)$, and thus low-latitude voltage, than the red and green traces. Conversely, during the predominantly positive $B_{T}$ sector $\left(\theta \simeq+90^{\circ}\right)$ between days $\sim 97$ and $\sim 107$, the green trace, representing the maximum positive 


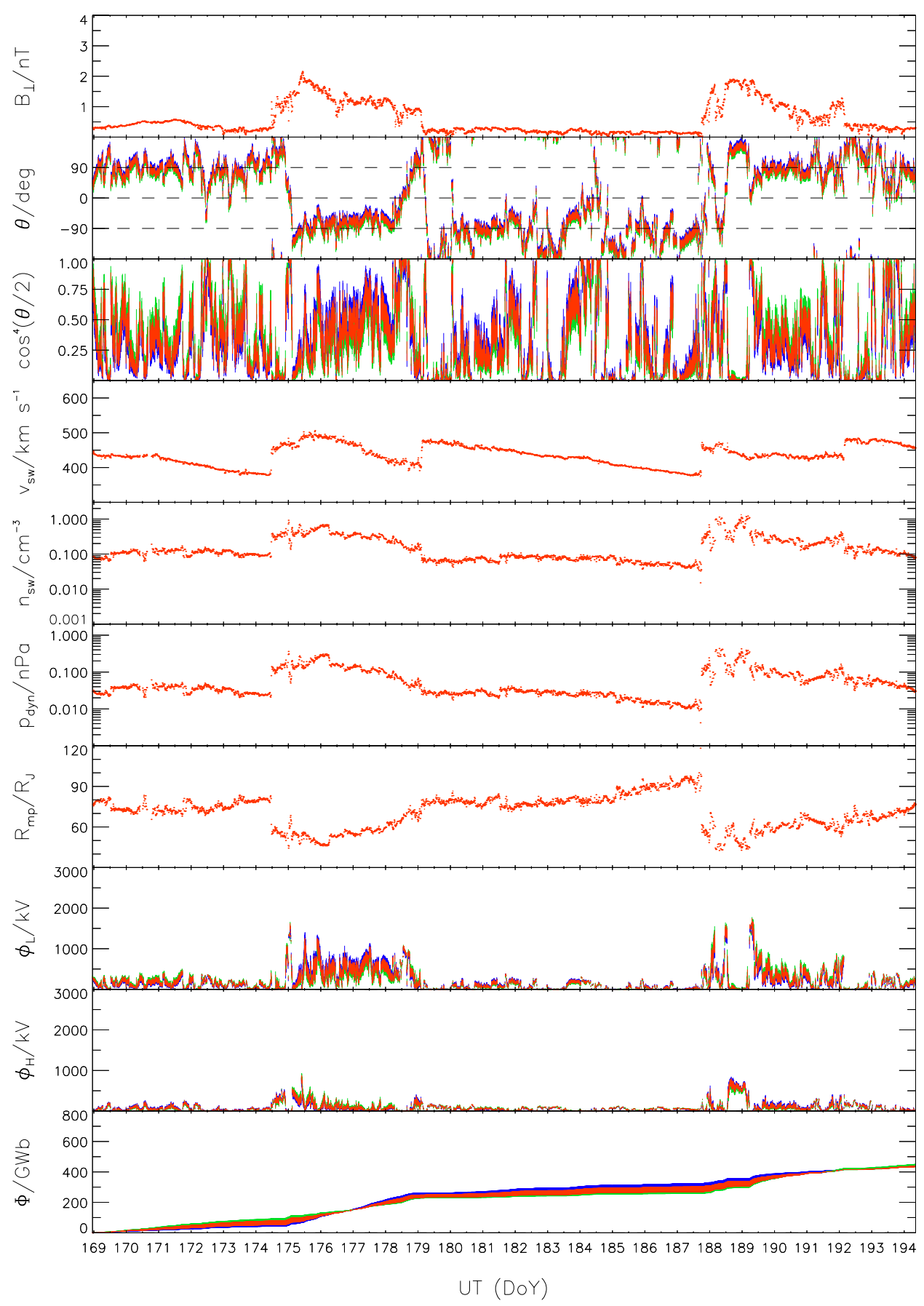

Fig. 6. As Fig. 3 for Ulysses data from the interval over days 168-194 in 2004.

angle of the spin axis to the $\mathrm{N}$-axis, exhibits the higher lowlatitude voltage, although as stated previously these variations are relatively small with respect to the overall modulation due to the changes in IMF strength and direction. However, whilst the above variations may be relatively small, the cumulative effect of the initial sustained period of negative IMF clock angle can be seen in the $\Phi$ panel by the gradual separation of the coloured data and the widening of the trace until day 97, with the blue line higher than the green. However, the reverse is the case in the following sector, such that the coloured lines then re-converge. Thus, if over the solar rotation as a whole there occurs approximately equal intervals of negative and positive $B_{T}$ sectors the total amount of open flux created is not greatly affected by the direction 
of Jupiter's spin axis. We should note that during the period shown in Fig. 3, Jupiter was in fact near to the spacecraft following the Jupiter flyby, and had an orbital longitude of $\sim 160^{\circ}$. Thus the spin axis had an angle of $\sim+3^{\circ}$ relative to the $\mathrm{N}$-axis, such that the actual values appropriate to the period lie roughly mid-way between the red and green traces. As a summary we show in Table 1 the values of the parameters that occur in Eq. (1) averaged over each solar rotation during this data epoch. The average amount of flux estimated to be created per solar rotation during the 1992 interval is $\sim 610 \mathrm{GWb}$, which corresponds to an average low-latitude voltage of $\sim 280 \mathrm{kV}$, with the values ranging between $\sim 520 \mathrm{GWb}$ and $\sim 800 \mathrm{GWb}$ per rotation, as shown. The averaged high-latitude voltages similarly vary between $\sim 90$ and $\sim 250 \mathrm{kV}$, with an overall average of $\sim 170 \mathrm{kV}$.

Turning now to the 1998 Ulysses data, representing the rising phase of the solar cycle, we have examined eight solar rotations of data spanning days 024-228. In Fig. 4 we show an example for the solar rotation during days 75-101. These data clearly suggest rather different solar wind conditions to those seen in 1992. The clock angle of the IMF switches often between $\sim \pm 90^{\circ}$, such that six "sectors" can be distinguished, and no large-amplitude stream interactions are evident. Instead the key event in terms of low-latitude magnetopause reconnection during this interval is the flux rope that passes over the spacecraft between $\sim 12: 00$ UT on day 87 and $\sim 12: 00$ UT on day 90 , characterised by an enhancement in the magnetic field to $\sim 3 \mathrm{nT}$ and a smooth $360^{\circ}$ rotation in the field direction. The average plasma velocity is $\sim 370 \mathrm{~km} \mathrm{~s}^{-1}$, lower than the $\sim 435 \mathrm{~km} \mathrm{~s}^{-1}$ average during the 1992 interval shown in Fig. 3, and the range of velocities of $\sim \pm 25 \mathrm{~km} \mathrm{~s}^{-1}$ is also less than the $\sim 140 \mathrm{~km} \mathrm{~s}^{-1}$ range in Fig. 3. The average number density, $\sim 0.3 \mathrm{~cm}^{-3}$, is similar to that in Fig. 3, and the range of variability is also similar. The low-latitude dayside reconnection voltage again exhibits a "background" of the order of a hundred $\mathrm{kV}$, which is punctuated by an enhancement event associated with the flux rope and surrounding structure. During this event the estimated low-latitude voltage is elevated to a peak of $\sim 2 \mathrm{MV}$, and $\sim 150 \mathrm{GWb}$ of open flux is estimated to be created during the two day period from 12:00 UT on day 87 to 12:00 UT on day 90 . Over the whole $\sim 5$ day period between days 86 and $91, \sim 300 \mathrm{GWb}$ is estimated to be created, i.e. over half of the $\sim 550 \mathrm{GWb}$ estimated to have been produced over the solar rotation as a whole. The high-latitude reconnection voltage does not exhibit any significant enhancement since, although the IMF briefly turned southward twice as the flux rope passed over the spacecraft, the field magnitude is not as high during these times as it was during the period of northward IMF. The parameter values occurring in Eq. (1), averaged throughout each of the eight solar rotations examined and the whole 1998 epoch, are again shown in Table 1 . The average amount of open flux that is created over the whole 1998 interval is $\sim 510 \mathrm{GWb}$ per solar rotation (corresponding to an average low-latitude voltage of $\sim 230 \mathrm{kV}$ ), with values ranging between $\sim 320 \mathrm{GWb}$ and $\sim 670 \mathrm{GWb}$. The averaged high-latitude voltage is $\sim 110 \mathrm{kV}$.
Figure 5 shows Cassini data between days 323 and 348 in 2000, which represent solar maximum conditions. Both magnetic field and plasma data during the Jupiter flyby period are, unfortunately, not continuous, such that the interval selected represents the most complete 25-day interval in terms of coverage from both magnetic field and plasma instruments together. The grey lines in the plasma data show the linear interpolation between the gaps in the plasma values. Again, the colours represent the variations due to Jupiter's spin and magnetic axis variations, as above, but in this case Jupiter was in the vicinity of the spacecraft when these data were taken and had a spin axis angle of $\sim+7^{\circ}$ relative to the $\mathrm{N}$-axis. Therefore the green trace is the one appropriate to actual conditions at Jupiter in this case. A clear compression-rarefaction structure can be now seen in the field magnitude and plasma velocity data, although the clock angle data are highly variable around the HCS crossings. The first compression region, which lasts from near the start of the interval shown to day $\sim 330$, contains large field magnitudes, up to $\sim 3.5 \mathrm{nT}$, and is accompanied by a corresponding large enhancement in the estimated voltages, up to $\sim 3 \mathrm{MV}$ and $\sim 1.5 \mathrm{MV}$ for low- and high-latitudes, respectively. There follows a rarefaction region of low field strength, of order a few tenths of a nT, and decreasing plasma velocity from $\sim 580 \mathrm{~km} \mathrm{~s}^{-1}$ to $\sim 400 \mathrm{~km} \mathrm{~s}^{-1}$, associated with low voltages of order a hundred $\mathrm{kV}$. A second, minor compression region begins on day 339 associated with the HCS crossing a day later. A region of enhanced field follows, beginning on day $\sim 343$, in which the estimated low- and highlatitude voltages are elevated to $\sim 2 \mathrm{MV}$ and $\sim 1 \mathrm{MV}$, respectively. Over this interval approximately $\sim 480 \mathrm{GWb}$ of open flux is estimated to have been produced, corresponding to an average low-latitude voltage of $\sim 220 \mathrm{kV}$. The average highlatitude voltage is $\sim 130 \mathrm{kV}$.

We finally consider the 2004 Ulysses interval, representing declining phase/solar minimum conditions, in which we examine eight solar rotations of data spanning days 092-295. In Fig. 6 we show an example for the solar rotation during days 168-194. The IMF clock angle indicates a two sector structure, with associated compression and rarefaction regions. Again the rarefaction regions are associated with a low "background" voltage, while the compressions are associated with enhancements. The maximum estimated voltages in the compression regions are relatively modest in this example, $\sim 1.6 \mathrm{MV}$ and $\sim 0.8 \mathrm{MV}$ for low- and high-latitudes respectively, reflecting the lower field strength than in the other intervals examined. The amount of open flux estimated to have been produced during this solar rotation is $\sim 430 \mathrm{GWb}$, corresponding to an average low-latitude voltage of $\sim 200 \mathrm{kV}$. The results for the other solar rotations in the 2004 interval are shown in Table 1, where it can be seen the average amount of open flux created per rotation is $\sim 410 \mathrm{GWb}$, corresponding to an average low-latitude voltage of $\sim 190 \mathrm{kV}$. The average high-latitude voltage is $\sim 110 \mathrm{kV}$.

With regard to the distribution of voltage values, we show in Fig. 7 a normalised distribution plot of the low-latitude reconnection voltage (specifically the middle values in the red 


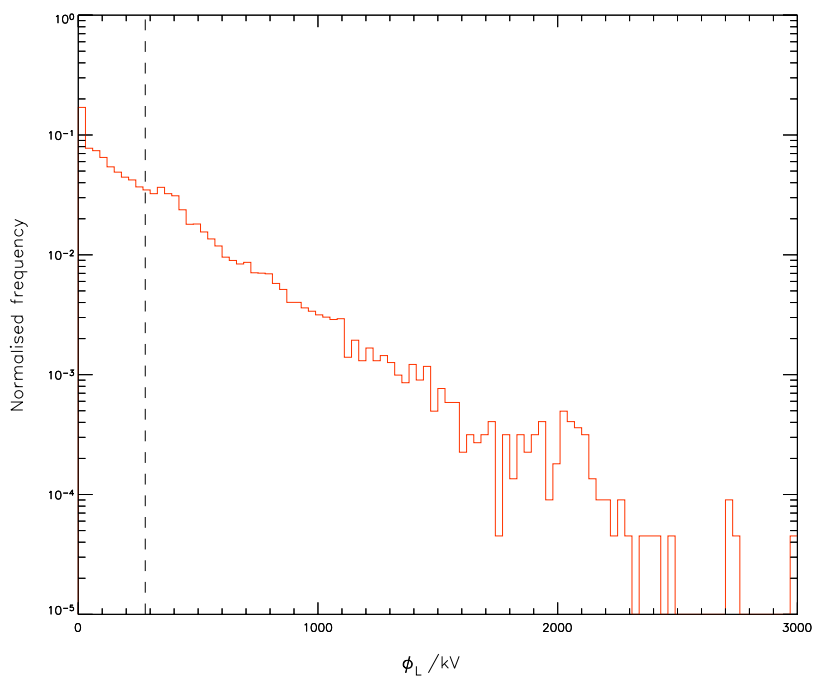

Fig. 7. Plot of the normalised distribution of low-latitude reconnection voltages derived from the entire 1992 Ulysses data set over days 008-161, shown on a linear-log scale. The values used are those from the middle of the red trace, i.e. the case when $\hat{S}_{S \perp}$ (i.e. the N-axis) and $\hat{\boldsymbol{M}}_{\boldsymbol{J} \perp}$ are co-aligned in the T-N plane. The vertical dashed line shows the location of the distribution mean.

trace) computed using the entire 1992 Ulysses data set over days 008-161 (omitting days 33-59), shown on a linear-log scale. The dashed vertical line shows the distribution mean of $279 \mathrm{kV}$. It can be seen that, despite the above discussions that might imply a "bimodal" distribution of voltages due to the prevalent background-enhancement region structure, the distribution is almost linear up to a voltage of $\sim 2 \mathrm{MV}$, implying that the probability simply depends exponentially on the reconnection voltage. The equivalent plots for the 1998 and 2004 data sets are essentially similar, except that the slopes are modestly steeper, reflecting the lower average voltage values during these intervals.

\section{Discussion and summary}

In this paper we have estimated the low- and high-latitude jovian magnetopause reconnection voltages using an extended interplanetary data set at $\sim 5 \mathrm{AU}$ and empirical formulas that have been validated at Earth and previously applied to Saturn. We have employed Ulysses and Cassini data from 22 solar rotations when the spacecraft were in the vicinity of Jupiter's orbit, and obtained time series of the reconnection voltages. We found that the average low- and highlatitude magnetopause reconnection voltages at Jupiter are of order $\sim 230 \mathrm{kV}$ and $\sim 130 \mathrm{kV}$, respectively. This value of the low-latitude reconnection voltage is approximately ten times that derived for Saturn by Jackman et al. (2004), yet is a factor of $\sim 4$ less than the $1 \mathrm{MV}$ estimate for Jupiter of Kennel and Coroniti (1977). However, a considerable amount of variation occurs within this average value. The "background" voltage of order $\sim 100 \mathrm{kV}$, associated with rarefaction regions, is generally punctuated by one or two events per solar rotation in which the magnetopause reconnection voltage is significantly elevated above the background. These events, in which the low-latitude voltage is enhanced to $\sim 1-3 \mathrm{MV}$, are due to periods of high strength and/or northward IMF in compression regions and flux ropes. The high-latitude voltage is similarly found to be enhanced, to $\sim 0.5-1.5 \mathrm{MV}$, when the IMF turns southward during these periods. Although the voltage across the magnetosphere as a whole associated with plasma corotation is $\sim 400 \mathrm{MV}$, as previously estimated by Brice and Ionannidis (1970) and Kennel and Coroniti $(1975,1977)$, the voltage across the middle magnetosphere current sheet region is much less than this. As stated in the introduction, Nichols and Cowley (2005) and Cowley et al. (2005) recently estimated the voltage due to sub-corotation between $\sim 20 \mathrm{R}_{\mathrm{J}}$ in the equatorial plane and the magnetopause to be $\sim 10 \mathrm{MV}$. Thus the Dungey cycle voltage, typically of order a few MV during enhancement events, is clearly not negligible compared with the corotation voltage across the middle and outer magnetospheres. The implication of these large voltages is that during enhancement events magnetopause reconnection may have a significant effect on an otherwise rotationally dominated magnetosphere. In addition, the probability distribution of reconnection voltage values appears to be exponentially dependant on the voltage, despite the apparent "bimodal" behaviour due to the rarefaction-enhancement region structure. We also considered the effect of the angle of Jupiter's spin and magnetic axes on the voltage values, as this varies on the time scales of both a jovian day and a jovian year. We found that when Jupiter's magnetic axis lies at a positive angle to the $\mathrm{N}$-axis (see Fig. 2), the IMF sector with positive $B_{T}$ exhibits higher estimated reconnection voltages than the sector with negative $B_{T}$, and vice versa. This effect, however, represents only a first order correction to the reconnection voltage, which is primarily modulated by the strength and direction of the IMF.

We also found that the average amount of open flux produced per solar rotation is $\sim 500 \mathrm{GWb}$, with individual intervals during a given epoch varying by factors of less than $\sim \pm 30 \%$, despite the interplanetary conditions varying considerably from interval to interval. However, the average value is found to depend on the solar cycle, ranging from an average of $\sim 600 \mathrm{GWb}$ per solar rotation in 1992 near solar maximum to $\sim 400 \mathrm{GWb}$ per solar rotation in 2004 near solar minimum. This result is consistent with that of Hapgood et al. (1991), who showed that the magnitude of the IMF is $50 \%$ higher at solar maximum than solar minimum at distances of $\sim 1 \mathrm{AU}$. The amount of open flux in each lobe of Jupiter's tail is estimated to be $\sim 300-500 \mathrm{GWb}$, corresponding to a field of $\sim 1-1.5 \mathrm{nT}$ in a tail of radius $200 \mathrm{R}_{\mathrm{J}}$ (e.g. Acuña et al., 1983). Thus, we estimate that it takes $\sim 15$ 25 days to produce enough open flux to replenish the tail. Following Dungey (1965), we multiply this time by a representative value of the solar wind speed, e.g. $450 \mathrm{~km} \mathrm{~s}^{-1}$, to obtain an estimate of the length of the magnetotail of $\sim 3.8$ 6.5 AU. This result is larger than that calculated by Kennel and Coroniti (1977) due to our smaller estimate of the 
average reconnection voltage, but our value does allow Saturn to pass through Jupiter's tail, and, like our voltage estimate itself, is expected to contain considerable variation on the time scale of a solar rotation.

Acknowledgements. J. D. Nichols and S. W. H. Cowley were supported during the course of this study by PPARC grant PPA/G/O/2003/00013. D. J. McComas and Ulysses/SWOOPS observations were supported by NASA. The authors would like to thank A. Balogh and M. K. Dougherty at Imperial College London for provision of Ulysses and Cassini magnetometer data, respectively, and F. J. Crary and the CAPS instrument team for use of the Cassini solar wind parameters employed in this study. CAPS is supported by a contract with NASA/JPL. We also thank the SIDC, Belgium, for use of sunspot data. J. D. Nichols would like to thank A. Grocott and E. Bunce for helpful discussions.

Topical Editor I. A. Daglis thanks N. Achilleos and another referee for their help in evaluating this paper.

\section{References}

Acuña, M. H., Behannon, K. W., and Connerney, J. E. P.: Jupiter's magnetic field and magnetosphere, in: Physics of the Jovian Magnetosphere, edited by: Dessler, A. J., Cambridge Univ. Press, Cambridge, U.K., 1983.

Balogh, A., Beek, T. J., Forsyth, R. J., Hedgecock, P. C., Marquedant, R. J., Smith, E. J,. Southwood, D. J., and Tsurutani, B. T.: The magnetic field investigation on the Ulysses mission: Instrumentation and preliminary scientific results , Astron. Astrophys. Suppl., 92 (2), 221-236, 1992.

Bame, S. J., McComas, D. J., Barraclough, B. L., Phillips, J. L., Sofaly, K. J., Chavez, J. C., Goldstein, B. E., and Sakurai, R. K.: The Ulysses solar wind plasma experiment, Astron. Astrophys. Suppl., 92 (2), 237-265, 1992.

Brice, N. M. and Ioannidis, G. A.: The magnetospheres of Jupiter and Earth, Icarus, 13, 173-183, 1970.

Bunce, E. J., Cowley, S. W. H., and Yeoman, T. K.: Jovian cusp processes: Implications for the polar aurora, J. Geophys. Res., 109, A09S13, doi:10.1029/2003JA010280, 2004.

Bunce, E. J., Cowley, S. W. H., and Milan, S. E.: Interplanetary magnetic field control of Saturn's polar cusp aurora, Ann. Geophys., 23, 1405-1431, 2005,

\section{SRef-ID: 1432-0576/ag/2005-23-1405}

Cowley, S. W. H. and Bunce E. J.: Origin of the main auroral oval in Jupiter's coupled magnetosphere-ionosphere system, Planet. Space Sci., 49, 1067-1088, 2001.

Cowley, S. W. H., Alexeev, I. I., Belenkaya, E. S., Bunce, E. J., Cottis, C. E., Kalegaev, V. V., Nichols, J. D., Prangé, R., and Wilson, F. J.: A simple axisymmetric model of magnetosphereionosphere coupling currents in Jupiter's polar ionosphere, J. Geophys. Res., 110, A11209, doi:10.1029/2005JA011237, 2005.

Delamere, P. A. and Bagenal, F.: Modeling variability of plasma conditions in the Io torus, J. Geophys. Res., 108(A7), 1276, doi:10.1029/2002JA009706, 2003.

Dougherty, M. K., Kellock, S., Southwood, D. J., Balogh, A., Smith, E. J., Tsurutani, B. T., Gerlach, B., Glassmeier, K.-H., Gleim, F., Russell, C. T., Erdos, G., Neubauer, F. M., and Cowley, S. W. H.: The Cassini magnetic field investigation, Space Sci. Rev., 114, 331-383, 2004.

Dungey, J. W.: The length of the magnetospheric tail, J. Geophys. Res., 70, 1753, 1965.
Fränz, M. and Harper, D.: Heliospheric coordinate systems, Planet. Space Sci., 50 (2), 217-233, 2002.

Gazis, P. R.: A large scale survey of corotating interaction regions and their successors in the outer heliosphere, J. Geophys. Res., 105, 19-34, 2000.

Gérard, J.-C., Bunce, E. J., Grodent, D., Cowley, S. W. H., Clarke, J. T., and Badman, S. V.: Signature of Saturn's auroral cusp: Simultaneous Hubble Space Telescope FUV observations and upstream solar wind monitoring, J. Geophys. Res., 110, A11201, doi:10.1029/2005JA011094, 2005.

Gosling, J. T. and Pizzo, V. J.: Formation and evolution of corotating interaction regions and their three dimensional structure, Space Sci. Rev., 89, 21-52, 1999.

Grodent, D., Clarke, J. T., Waite Jr., J. H., Cowley, S. W. H., Gérard, J.-C., and Kim, J.: Jupiter's polar auroral emissions, J. Geophys. Res., 108(A10), 1366, doi:10.1029/2003JA010017, 2003.

Grodent, D., Gérard, J.-C., Clarke, J. T., Gladstone, G. R., and Waite Jr., J. H.: A possible auroral signature of a magnetotail reconnection process on Jupiter, J. Geophys. Res., 109, A05201, doi:10.1029/2003JA010341, 2004.

Hapgood, M. A., Lockwood, M., Bowe, G. A., and Willis, D. M.: Variability of the interplanetary medium at $1 \mathrm{AU}$ over 24 years: 1963-1986, Planet. Space Sci., 39, 3, 411-423, 1991.

Hill, T. W.: Inertial limit on corotation, J. Geophys. Res., 84, 6554 6558, 1979.

Hill, T.W.: The jovian auroral oval, J. Geophys. Res., 106, 81018108, 2001.

Huddleston, D.E., Russell, C.T., Kivelson, M.G., Khurana, K.K., and Bennett, L.: Location and shape of the jovian magnetopause and bow shock, J. Geophys. Res., 103, 20075-20082, 1998.

Jackman, C. M., Achilleos, N., Bunce, E. J., Cowley, S. W. H., Dougherty, M. K., Jones, G. H., Milan, S. E., and Smith, E. J.: Interplanetary magnetic field at $\sim 9 \mathrm{AU}$ during the declining phase of the solar cycle and its implications for Saturn's magnetospheric dynamics, J. Geophys. Res., 109, A11203, doi:10.1029/2004JA010614, 2004.

Kennel, C. F. and Coroniti, F. V.: Is Jupiter's magnetosphere like a pulsar's or Earth's?, in: The magnetospheres of Earth and Jupiter, edited by: Formisano, V., D. Reidel, Norwell, Mass., 451, 1975.

Kennel, C. F. and Coroniti, F. V.: Possible origins of time variability in Jupiter's outer magnetosphere 2: Variations in solar wind magnetic field, Geophys. Res. Lett., 4, 215-218, 1977.

Kunow, H.: Solar Wind: Corotating interaction regions, in: Encyclopaedia of Astronomy and Astrophysics, Institute of Physics Publ., Bristol, 4, 2825, 2001.

Lepping, R. P., Desch, M. D., Klein, L. W., Sittler Jr., E. C., Sullivan, J. D., Kurth, W. S., and Behannon, K. W.: Structure and other properties of Jupiter's distant magnetotail, J. Geophys. Res., 88, 8801-8815, 1983.

McComas, D. J., Goldstein, R., Gosling, J. T., and Skoug, R. M.: Ulysses' second orbit: Remarkably different solar wind, Space Sci. Rev., 97, 99-103, 2001.

Milan, S. E., Cowley, S. W. H., Lester, M., Wright, D. M., Slavin, J. A., Fillingim, M., Carlson, C. W., and Singer, H. J: Response of the magnetotail to changes in the open flux content of the magnetosphere, J. Geophys. Res., 109, A04220, doi:10.1029/2003JA010350, 2004.

Nichols, J. D. and Cowley, S. W. H.: Magnetosphere-ionosphere coupling currents in Jupiter's middle magnetosphere: Effect of magnetosphere-ionosphere decoupling due to field-aligned auroral voltages, Ann. Geophys., 23, 799-808, 2005,

SRef-ID: 1432-0576/ag/2005-23-799. 
Nishida, A. and Maezawa, K.: IMF control and convection in the jovian magnetosphere, J. Geomag. Geoelectr., 38, 741-757, 1986.

Pallier, L. and Prangé, R.: More about the structure of the high latitude jovian aurorae, Planet. Space Sci., 49, 1159-1173, 2001.

Perrault, P. and Akasofu, S.-I.: A study of geomagnetic storms, Geophys. J. R. Astron. Soc., 54, 547-573, 1978.

Pontius Jr., D. H.: Radial mass transport and rotational dynamics, J. Geophys. Res., 102, 7137-7150, 1997.

Southwood, D. J. and Kivelson, M. G.: A new perspective concerning the influence of the solar wind on Jupiter, J. Geophys. Res., 106, 6123-6130, 2001.

Vasyliunas, V. M.: Plasma distribution and flow, in: Physics of the Jovian Magnetosphere, edited by: Dessler, A. J., Cambridge Univ. Press, Cambridge, U.K., 395, 1983.
Young, D. T., Berthelier, J. J., Blanc, M., Burch, J. L., Coates, A. J., Goldstein, R., Grande, M., Hill, T. W., Johnson, R. E., Kelha, V., McComas, D. J., Sittler, E. C., Svenes, K. R., Szego, K., Tanskanen, P., Ahola, K., Anderson, D., Bakshi, S., Baragiola, R. A., Barraclough, L., Black, R. K., Bolton, S., Booker, T., Bowman, R., Casey, P., Crary, F. J., Delapp, D., Dirks, G., Eaker, N., Funsten, H., Furman, J. D., Gosling, J. T., Hannula, H., Holmlund, C., Huomo, H., Illiano, J. M., Jensen, P., Johnson, M. A., Linder, D. R., Luntama, T., Maurice, S., McCabe, K. P., Mursula, K., Narheim, B. T., Nordholt, J. E., Preece, A., Rudzki, J., Ruitberg, A., Smith, K., Szalai, S., Thomsen, M. F., Viherkanto, K., Vilppola, J., Vollmer, T., Wahl, T. E., Wuest, M., Ylikorpi, T., and Zinsmeyer, C.: : Cassini plasma spectrometer investigation, Space Sci. Rev., 114, 1-112, 2004. 